\title{
Anticoagulação na gravidez
}

\author{
Flávia C. dos Santos, ${ }^{1 *}$ Guilherme R. de Jesús ${ }^{1}$, Nilson R. de Jesús, ${ }^{1}$ Roger A. Levy ${ }^{2}$
}

\section{Resumo}

As mudanças anatômicas e hormonais que ocorrem no organismo da mulher tornam as gestantes suscetíveis, expostas aos riscos de novo evento trombótico durante a gravidez. Dessa forma, é importante sabermos atuar na profilaxia e termos conhecimento do uso da anticoagulação em situações especiais e em patologias em que a trombose pode ocorrer na ausência de um manejo adequado. Nessas ocasiões, a escolha do anticoagulante se transforma em um grande desafio. Os cumarínicos são drogas que atuam na inibição da síntese dos fatores de coagulação. São considerados teratogênicos quando utilizados no primeiro trimestre e exigem controle pelo índice internacional de normatização(INR), porém, têm a vantagem do baixo custo e da administração por via oral, podendo ser uma alternativa quando utilizado entre a $13^{\mathrm{a}}$ e a $36^{a}$ semana de gestação. As heparinas atuam como cofator da antitrombina III, aumentando sua capacidade de neutralizar enzimas hemostáticas. Requerem administração por via parenteral por serem inativadas no intestino. Quando usadas por longos períodos, podem causar osteopenia e trombocitopenia. Com relação às medicações alternativas, não existem muitos estudos que possam garantir a eficácia e a segurança de sua utilização durante a gravidez. Ficam reservadas para os casos de contraindicação ao uso da heparina. A anticoagulação nas pacientes portadoras de síndrome antifosfolipídeo (SAF) é feita de acordo com as manifestações da doença que podem variar de perdas gestacionais associadas ou não a tromboses arteriais ou venosas. Com relação às pacientes portadoras de trombofilias hereditárias, a anticoagulação na gravidez será feita tomando como base o tipo de trombofilia (baixo ou alto risco) e a ocorrência ou não de evento trombótico prévio. As pacientes portadoras de válvula cardíaca metálica devem ser submetidas à anticoagulação plena durante toda a vida e o cumarínico é mais efetivo que a heparina na prevenção de complicações tromboembólicas nessas pacientes.

Descritores: Gravidez; Heparina; Trombofilia.

\section{Abstract}

\section{Anticoagulation in pregnancy}

The anatomical and hormonal changes that occur in a woman's body become susceptible pregnant women at risk of new thrombotic event during pregnancy. Thus, it is important that we know work in terms prophylaxis and knowledge of the use of anticoagulation in special situations and conditions in which thrombosis be present in the absence of proper management. On these occasions the choice of anticoagulant becomes a challenge. The coumarin are drugs that act by
1. Departamento Ginecologia e Obstetrícia. Faculdade de Ciências Médicas. Universidade do Estado do Rio de Janeiro. Rio de Janeiro, RJ, Brasil.

2. Departamento de Clínica Médica. Faculdade de Ciências Médicas. Universidade do Estado do Rio de Janeiro. Rio de Janeiro, RJ, Brasil.

*Endereço para correspondência:

Núcleo Perinatal, HUPE, UERJ

Av. Professor Manoel de Abreu, 500, 1ㅇandar

Rio de Janeiro, RJ, Brasil. CEP 20550-170.

E-mail: flavviacs@yahoo.com.br

Revista HUPE, Rio de Janeiro, 2015;14(2):71-77

doi: 10.12957/rhupe.2015.18442

Recebido em 01/12/2014. Aprovado em 22/12/2014.

inhibiting the synthesis of coagulation factors. Are considered teratogenic when used in the first quarter and demand control by international standardization ratio (INR), however, have the advantage of low cost and oral administration maybe an alternative when used between the 13[th] and the 36[th] week of pregnancy. Heparins act as a cofactor for antithrombin III enhancing their ability to neutralize hemostatic enzymes. Require parenteral administration because they are inactivated in the intestines. When used for extended periods can cause osteopenia and thrombocytopenia. With respect to alternative medications there are not many studies that can ensure the efficacy and safety of its use during pregnancy. They are reserved for cases of contraindication to the use of heparin. The anticoagulation in patients with APS is made in accordance with the manifestations of the disease which can vary from pregnancy losses associated or not with arterial or venous thromboses. With respect to patients with hereditary thrombophilia, anticoagulation will be made based on the type of trombifilia (low or high risk) and the occurrence of previous thrombotic event. The patients with metal heart valve must undergo anticoagulation throughout life and the coumarin is more effective than heparin in the prevention of thromboembolic complications in these patients.

Keywords: Pregnancy; Heparina; Thrombophilia.

\section{Resumen}

\section{La anticoagulación en el embarazo}

Los cambios anatómicos y hormonales que se producen en el organismo de la mujer hacen que se vuelvan vulnerables, expuestas a los riesgos de un nuevo evento trombótico durante el embarazo. Por lo tanto, es importante saber qué 
hacer para la profilaxis y tener conocimiento del uso de la anticoagulación en situaciones especiales y en patologías en las que la trombosis se puede producir por la ausencia de un manejo adecuado. En estas ocasiones la elección de anticoagulantes se convierte en un gran desafío. Los cumarínicos son fármacos que actúan en la inhibición de la síntesis de los factores de coagulación. Se consideran teratogénicos cuando se utilizan en el primer trimestre y exigen el control del índice internacional de normalización (INR), sin embargo, tienen la ventaja del bajo costo y de la administración por vía oral, pudiendo ser una alternativa usados entre la $13^{\mathrm{a}}$ y la $36^{\mathrm{a}}$ semana de embarazo. Las heparinas actúan como cofactor de la antitrombina III, aumentando su capacidad de neutralizar enzimas hemostáticas. Requieren administración por vía parenteral, ya que se inactivan en los intestinos. Cuando se utilizan durante largos periodos, pueden causar osteopenia

\section{Introdução}

O uso de anticoagulantes durante a gravidez está indicado às pacientes que apresentam suspeita ou diagnóstico de trombose venosa profunda, embolia pulmonar, fibrilação atrial crônica, dilatação atrial; na profilaxia de trombose nas portadoras de prótese valvares metálicas e em pacientes com eventos recentes de tromboses arteriais e acidente vascular cerebral (AVC). ${ }^{1}$ Atualmente, esse grupo está acrescido das portadoras da síndrome antifosfolipídeo (SAF) e de outras trombofilias adquiridas ou hereditárias. ${ }^{2}$ Devido à possibilidade dos anticoagulantes orais causarem embriopatias e hemorragia fetal e as heparinas apresentarem menor eficácia em pacientes portadoras de válvulas metálicas, a anticoagulação na gravidez apresenta diversas controvérsias.

Todas as gestantes em uso de anticoagulantes devem ser informadas da importância da correta adesão ao tratamento, dos seus riscos, da interação com drogas e alimentos, de como fazer o controle e atuar em situações de emergência e receber cartão ou formulário de controle dos exames. No cartão de acompanhamento do pré-natal, a informação "em uso de anticoagulante" deve estar registrada na primeira página.

\section{Anticoagulantes orais}

Os cumarínicos bloqueiam a síntese hepática de fatores da coagulação e de anticoagulantes (proteína C e S) dependentes de vitamina K. Uma vez que a ação da droga é baseada principalmente na inibição da síntese de fatores da coagulação, são necessários de um a três dias para que a droga se torne efetiva. O bloqueio y trombocitopenia. Con respecto a las medicaciones alternativas, no hay muchos estudios que permitan garantizar la eficacia y seguridad de su utilización durante el embarazo. Se reservan para los casos de contraindicación en el uso de la heparina. La anticoagulación en pacientes portadoras de SAF se hace de acuerdo con las manifestaciones de la enfermedad que pueden variar de pérdidas gestacionales asociadas o no con las trombosis arteriales o venosas. Con respecto a los pacientes portadores de trombofilias hereditarias, la anticoagulación en el embarazo se hará sobre la base del tipo de trombofilia (bajo o alto riesgo) y la presencia o no de evento trombótico previo. . Las pacientes portadoras de válvula cardiaca metálica deben someterse a la anticoagulación plena durante toda la vida y el cumarínico es más efectivo que la heparina en la prevención de complicaciones tromboembólicas en estas pacientes.

Palabras clave: Embarazo; Heparina; Trombofilia.

da ação da proteína C e S antecede ao dos fatores da coagulação, resultando em efeito trombogênico no início do tratamento. Tal fato torna obrigatório o uso concomitante de heparina não fracionada (HNF) ou heparina de baixo peso molecular (HBPM), ambas por via subcutânea nos dois ou três primeiros dias antes do início do uso do cumarínico, e essa só deve ser suspensa quando o alvo do INR for alcançado ou estiver próximo. São totalmente absorvidos pelo trato gastrintestinal, o que possibilita o uso da medicação por via oral e aumenta a adesão da paciente.

As pacientes em idade fértil em uso de cumarínico devem ser orientadas a programar a gestação. O cumarínico é considerado teratogênico quando utilizado no primeiro trimestre. A incidência de embriopatias gira em torno de $4 \%$ a $6 \%$ e incluem hipoplasia ou agenesia do septo nasal, micrognatia, chondrodysplasiapunctacta, agenesia do corpo caloso e meningocele. ${ }^{3}$ Estudo multicêntrico prospectivo envolvendo 666 gestantes expostas a antagonistas da vitamina $\mathrm{K}$ observou que a embriopatia ocorreu em $0,6 \%$ dos casos, embora o risco tenha sido quatro vezes maior. ${ }^{4}$ Durante o segundo e terceiro trimestres, hemorragias podem causar microftalmia, atrofia óptica e malformação de Dandy-Walker (dilatação do quarto ventrículo, agenesia ou hipoplasia do vermis cerebelar e, frequentemente, hidrocefalia). ${ }^{5} \mathrm{~A}$ ocorrência dessas malformações está descrita apenas em relatos isolados de casos. As malformações relacionadas ao uso de cumarínicos na gestação, que configuram a chamada "síndrome warfarinica fetal" são mais frequentes quando há exposição fetal após a $6^{\mathrm{a}}$ semana de gestação. ${ }^{6}$

Após a $14^{a}$ semana de gestação, é possível o uso de 
cumarínico. Esse esquema de tratamento surgiu como uma alternativa ao uso das heparinas de baixo peso molecular, que têm um custo elevado, mas a paciente deve ser informada sobre os riscos fetais associados ao uso do cumarínico após o primeiro trimestre e fazer tal opção após adequado esclarecimento.?

No primeiro trimestre e nas quatro últimas semanas, o anticoagulante oral é substituído pela heparina. A dose inicial deve ser de $5 \mathrm{mg}$ por dia, tomados geralmente ao fim da tarde (18h), tempo suficiente para que os resultados dos exames já estejam disponíveis. Os objetivos de alargamento do tempo e atividade da protrombina (TAP) tomando como base o índice internacional de normatização (INR) é, para a maioria das pacientes, entre 2 e 3 e para algumas pacientes de alto risco entre 2,5 e 3,5. Os exames de controle devem ser solicitados cinco dias após o início da droga ou ajuste realizados. O controle da anticoagulação com cumarínico pode ser orientado pela escala proposta por um dos autores (Tabela 1).

A presença de sangramentos (ex.: gengivorragia, hematúria, epistaxe etc.) ou INR maior ou igual a 9 são indicações de internação. Se houver necessidade de reversão rápida da anticoagulação (sangramento ou necessidade de cirurgia de urgência), deve-se prescrever 10 mg IV de vitamina K (infusão em dez minutos) e duas unidades de plasma fresco congelado. Nesses casos deve-se repetir o TAP a cada seis horas. Nas pacientes portadoras de SAF, devido ao risco de estimular trombose em vários sítios do organismo, deve-se evitar o uso da vitamina K e utilizar somente plasma fresco. Quando estiver indicada a substituição do anticoagulante oral por heparina, o cumarínico pode ser suspenso imedia-

Tabela 1. Escala de Jesús para controle da anticoagulação com o uso do cumarínico.

\begin{tabular}{|c|c|c|}
\hline INR & AJUSTE & EXAMES \\
\hline$<1,2$ & 个 2mg na dose diária & 5 dias \\
\hline $1,2-2,0$ & 个 1mg na dose diária & 5 dias \\
\hline $2,0-3,0$ & Ideal & $\begin{array}{c}1 \mathrm{x} / \text { semana } \\
2 \mathrm{x} \text { no alvo }=15 / 15 \text { dias }\end{array}$ \\
\hline $3,0-5,0$ & $\begin{array}{l}\text { Suspender por } 1 \text { dia e } \\
\downarrow 1 \mathrm{mg} \text { na dose diária }\end{array}$ & 5 dias \\
\hline$>5,0$ & $\begin{array}{l}\text { Suspender e } \\
\text { reiniciar com } \downarrow 1 \mathrm{mg} \\
\text { após exames }\end{array}$ & $\begin{array}{l}\text { Diários até retornar } \\
\text { ao alvo }\end{array}$ \\
\hline
\end{tabular}

tamente e a heparina iniciada logo em seguida.

A ocorrência de desajustes súbitos e/ou intensos sinaliza a necessidade de investigar erros no entendimento da paciente da dose prescrita, desvios alimentares ou uso de medicações que interferem na ação do anticoagulante oral.

Existem alimentos que inibem a ação dos cumarínicos porque contêm grandes quantidades de vitamina K e podem alterar o INR em um usuário de cumarínico. Esses alimentos não são proibidos, mas devem ser ingeridos de uma forma "monótona" (quantidades diárias regulares e constantes) para que a dose do anticoagulante seja ajustada de acordo com a dieta.

\section{Heparina}

As heparinas previnem a formação e limitam a extensão do trombo. Atuam como um cofator da antitrombina III, aumentando sua capacidade de neutralizar enzimas hemostáticas. ${ }^{8}$ São inativadas no intestino e, por isso, requerem administração por via parenteral, tornando-se uma desvantagem quando o uso prolongado é necessário. As HNF possuem elevado peso molecular (em média $15.000 \mathrm{Da}$ ) e não atravessam a barreira placentária. ${ }^{9}$ Estas possuem meia-vida muito curta (4-6 horas) e biodisponibilidade de $10 \%$. $\mathrm{O}$ uso prolongado durante a gestação predispõe à formação de equimoses frequentes e infecções locais, e o desenvolvimento de osteopenia em um terço das pacientes. ${ }^{10}$ A trombocitopenia é outra complicação do uso da heparina não fracionada. A forma leve da trombocitopenia induzida pela heparina surge nos primeiros dias do uso da medicação e é reversível. A forma grave, imune e mais rara pode paradoxalmente causar trombose. ${ }^{3}$ Deve-se suspender a utilização da droga quando a contagem de plaquetas está abaixo de 50.000. Da mesma forma que as HNF, as HBPM não atravessam a barreira placentária, entretanto, apresentam como desvantagem o alto custo e como vantagem a meia-vida mais longa (12-24 horas), a biodisponibilidade de 85\% e o menor risco de trombocitopenia e sangramento. A enoxaparina e a dalteparina são as únicas HBPM, até o momento, amplamente avaliadas na gravidez. Tanto as HNF quanto as HBPM podem ser utilizadas de forma profilática ou terapêutica.

O uso profilático da heparina é indicado para pacientes que serão submetidas à grande cirurgia, ou qualquer cirurgia em pacientes com passado de tromboembolismo, obesidade mórbida e ainda para as restritas ao leito. As heparinas, nas doses profiláticas, são feitas 


\section{Artigo de revisão}

por via subcutânea. A dose de HNF é de 5.000 unidades a cada 12 horas. $^{11}$

Com relação ao uso terapêutico, procede-se com a heparinização plena, que pode ser feita por via intravenosa (IV) com HNF e por via subcutânea com HNF ou HBPM. A via subcutânea está indicada nas pacientes portadoras de TVP, prótese valvar mecânica, SAF ou outra trombofilia. A dose inicial da HNF é de 24.000 a $30.000 \mathrm{U}$, divididas em duas a quatro tomadas. O objetivo terapêutico é alargar o PTT 1,5 a duas vezes o PTT basal. Os exames de controle são solicitados uma a duas vezes por dia até atingir alvo e, a seguir, semanalmente. As amostras de sangue devem ser coletadas sempre seis horas após a última dose subcutânea. A dose recomendada da enoxaparina é de $1 \mathrm{mg} / \mathrm{kg} / \mathrm{dose}$ e da dalteparina de $100 \mathrm{UI} / \mathrm{kg} /$ dose, ambas repetidas a cada 12 horas. $^{1}$

A reversão da anticoagulação é feita com 50 mg de protamina por via intravenosa. Cada $1 \mathrm{mg}$ da protamina reverte $100 \mathrm{U}$ de heparina. Nas pacientes com SAF, devido aos mesmos motivos quando em uso de cumarínico, a reversão da anticoagulação com heparina não deve ser medicamentosa. O plasma fresco congelado deve ser o tratamento de escolha.

A via IV com HNF está indicada nos casos de trombose venosa profunda extensa (TVP), tromboembolismo pulmonar (TEP), AVE isquêmico, trombose valvar ou fibrilação atrial aguda. A heparinização IV baseada no peso da paciente é prescrita segundo as orientações da escala de Raschke (Tabela 2).

A dose de ataque é de $80 \mathrm{U} / \mathrm{kg}$ IV seguida de infusão contínua de $18 \mathrm{U} / \mathrm{kg} /$ hora $^{9}{ }^{9}$

Tabela 2. Escala de Raschke para controle da anticoagulação IV com o uso da heparina não fracionada.

\begin{tabular}{|c|c|c|}
\hline PTT - relação & PTT - tempo & AJUSTE \\
\hline$<1,2$ & $<35$ seg. & $\begin{array}{c}\text { Repetir dose de ataque } \\
\uparrow 4 \mathrm{U} / \mathrm{kg} / \mathrm{hora}\end{array}$ \\
\hline $1,2-1,5$ & $35-45$ seg. & $\begin{array}{l}\text { Bolus 40U/kg } \\
\uparrow 4 U / \mathrm{kg} / \text { hora }\end{array}$ \\
\hline $1,5-2,3$ & $45-70$ seg. & IDEAL \\
\hline $2,3-3,0$ & $71-90$ seg. & $\downarrow 2 U / k g / h o r a$ \\
\hline \multirow[t]{2}{*}{$>3,0$} & $90-120$ seg. & $\begin{array}{c}\text { Suspender } 1 \text { hora } \\
\text { Reiniciar } \downarrow 3 U / \mathrm{kg} / \text { hora }\end{array}$ \\
\hline & > 120 seg. & $\begin{array}{c}\text { Suspender } 1 \text { hora } \\
\text { Reiniciar com } 1 / 2 \text { dose }\end{array}$ \\
\hline
\end{tabular}

\section{Rotina de solicitação dos exames:}

a. obter PTT de seis em seis horas nas primeiras 24 horas e reajustar a dose conforme o resultado;

b. PTT de oito em oito horas até obter três resultados consecutivos ideais;

c. após os três resultados ideais: PTT uma vez ao dia (manhã);

d. qualquer necessidade de ajuste, voltar ao passo b.

Solução-padrão: soro glicosado 5\% - $245 \mathrm{ml}+5 \mathrm{ml}$ de HNF 5.000 U/ml - IV em bomba infusora. A solução em SG 5\% deve ser trocada de oito em oito horas e a solução em SF 0,9\% não necessita ser trocada até o final.

\section{Medicamentos alternativos}

Além dos anticoagulantes tradicionais, há possibilidade de uso de medicamentos alternativos.

\section{Danaparoide}

Da mesma forma que as HNF e HBPM, danaparoide catalisa a inativação do factor Xa mediada pela antitrombina. É administrado por via intravenosa e tem baixa permeabilidade placentária. Não é medicação de primeira escolha em gestantes por não haver estudos de boa qualidade e a sua utilização fica reservada aos casos de trombocitopenia induzida pela heparina que ocorre antes ou durante a gravidez.1,12,13

\section{Fondaparinux}

É um pentassacárido, inibidor selectivo do factor $\mathrm{Xa}$, que imita a ligação da heparina ao sítio ativo da antitrombina. Fondaparinux é administrado por via subcutânea. Dados da utilização dessa droga durante a gravidez são limitados e dados sobre a sua passagem através da placenta são controversos. O American College of Chest Physicians sugere que o seu uso durante a gravidez fique limitado aos casos em que há severa reação à heparina, como trombocitopenia e o uso do danaparoide seja inviável. ${ }^{1}$

\section{Argatroban}

É um inibidor direto da trombina que requer administração intravenosa contínua e monitoração pelo tempo da tromboplastina parcial ativada. Estudos em animais têm demonstrado a presença da medicação no leite.É rotulado pela FDA como categoria B na gravidez. Reservado aos casos com reação severa à heparina e que não podem receber danaparoide nem fondaparinux por reações alérgicas ou dados indisponíveis. ${ }^{14-16}$ 


\section{Agentes antitrombóticos}

O inibidor oral direto da trombina (dabigratan) e os inibidores orais do fator Xa - rivaroxabano, apixaban e edoxaban - não são usados durante a gravidez por ausência de informações sobre eficácia e segurança fetal. ${ }^{1}$

\section{Manejo durante o trabalho de parto e parto}

Nas pacientes que se encontram em uso de cumarínico, o mesmo deve ser trocado após 36 semanas de gestação por heparina, que será reiniciada após o procedimento e mantida até dois dias após, quando o cumarínico é reintroduzido. A HNF deve ser suspensa quando a paciente entrar na fase ativa do trabalho de parto. Nos casos de cesariana eletiva, ela deve ser realizada na $39^{a}$ semana de gestação e a HBPM deve ser suspensa 24 horas antes do parto. ${ }^{1}$ Caso a paciente entre em trabalho de parto em vigência de anticoagulação, seja profilática ou plena, a medicação deve ser suspensa para o acompanhamento do trabalho de parto, sem necessidade da prescrição de inibidores da ação da heparina.

\section{Manejo durante o puerpério e aleitamento}

O pós-parto é o período de maior risco de trombose. O reinício da anticoagulação deve ser feito com HBPM seis horas após o parto vaginal e 12 horas após a cesariana. O cumarínico pode ser iniciado 48 horas após a heparina, mantendo a administração simultânea dos anticoagulantes até que o INR atinja os valores esperados. Pacientes em uso de heparina profilática durante a gravidez devem manter o tratamento por seis semanas após o nascimento e, nestes casos, como não há necessidade do uso prolongado de anticoagulantes, sugerimos a utilização da HBPM durante todo o período. Essa opção é mais confortável para a paciente, uma vez que não há necessidade da repetição dos testes de coagulação para ajustes de doses, procedimentos obrigatórios se optarmos pelo uso do cumarínico. As pacientes portadoras de SAF clínica (trombose prévia ou acidente vascular encefálico) devem manter a anticoagulação por toda a vida e substituir a heparina pelo cumarínico, queé eliminado em quantidades mínimas no leite materno e, portanto, seguro para uso durante a amamentação, assim como a heparina e a aspirina..$^{17,18}$ O cumarínico deve ser administrado em doses diárias para manter o TAP com o INR entre 2,0 e 3,0 para as pacientes com eventos venosos e entre 2,5 e 3,5 para aquelas com eventos arteriais anteriores.

No momento do parto, as recomendações de anticoagulação para pacientes portadoras de trombofilias hereditárias não diferem das recomendações para outras trombofilias. ${ }^{1,2}$

\section{Anticoagulação em situações específicas}

A anticoagulação nas pacientes portadoras de SAF apresenta particularidades que estão relacionadas às manifestações da doença, que podem variar de perdas gestacionais associadas ou não a tromboses arteriais ou venosas.

\section{Síndrome antifosfolipídeo (SAF)}

Para pacientes com SAF e com história de abortamento recorrente, $o$ tratamento com aspirina em baixa dose (100 mg por dia) e heparina profilática aumenta, consideravelmente, a taxa de nascidos vivos..$^{17} \mathrm{~A}$ heparina interfere na cascata de coagulação, diminui a ativação do complemento, pode influenciar o mecanismo de placentação e evita a perda gestacional mediada pelos anticorpos antifosfolipídicos. ${ }^{19}$

Para pacientes com SAF e perda fetal tardia, recomendamos a aspirina em combinação com doses profiláticas de heparina, sendo eficazes tanto a HNF quanto a HBPM. ${ }^{17}$

Nas pacientes com história de trombose e presença persistente de anticorpos antifosfolipídeos (aPL), existe alto risco de recorrência da trombose. Assim, todas as mulheres com SAF e trombose anterior devem manter o tratamento antitrombótico durante todo o período de gravidez, bem como no pós-parto. O tratamento de escolha é a combinação de aspirina em baixa dose e heparina em dose plena para anticoagulação terapêutica. ${ }^{17}$ Não há evidência do tipo ideal de heparina: HNF ou HBPM. ${ }^{20}$ A HBPM, enoxaparina na dose de $1,0 \mathrm{mg} / \mathrm{kg}$ a cada 12 horas em injeções subcutâneas, possui menor risco de induzir trombocitopenia e não há necessidade do monitoramento através de testes de coagulação. ${ }^{1,10}$

\section{Trombofilias hereditárias}

Com relação às pacientes portadoras de trombofilias hereditárias, a anticoagulação na gravidez também será feita baseada no tipo de trombofilia (de baixo ou alto risco) e na manifestação clínica, ou seja, na ocorrência e gravidade do evento trombótico., ${ }^{1,2}$

As pacientes com história prévia de TEV ou que 


\section{Artigo de revisão}

apresentem um evento agudo durante a gravidez deverão receber anticoagulação durante a gestação e puerpério, independentemente do diagnóstico de trombofilia hereditária. ${ }^{1}$ As pacientes com trombofilia hereditária, mas sem história pessoal de TEV, deverão receber profilaxia de acordo com o tipo de trombofilia e com a história familiar de TEV.1,2 As trombofilias consideradas de baixo risco são as mutações heterozigóticas do fator V ou do gene da protrombina G20210A e as deficiências da proteína C ou da proteína S. Enquanto as trombofilias de alto risco são as mutações homozigóticas do fator V ou do gene da protrombina G20210A, deficiência da antitrombina III e duplos heterozigotos para fator V e para o gene da protrombina G20210A. ${ }^{2}$

\section{Prótese cardíaca metálica}

As pacientes portadoras de válvula cardíaca metálica devem ser submetidas à anticoagulação plena durante toda a vida para a prevenção de trombo valvar, falência valvar e trombose sistêmica. Na gravidez pode ser utilizado tanto o cumarínico como a heparina, seja ela HNF ou HBPM. O cumarínico é mais efetivo que a heparina na prevenção de complicações tromboembólicas nessas pacientes. ${ }^{21} \mathrm{O}$ protocolo da European Society of Cardiology recomenda anticoagulação com varfarina no segundo e terceiro trimestres da gestação. O uso no primeiro trimestre pode ser considerado, se a dose do cumarínico for menor que $5 \mathrm{mg} .{ }^{22}$ Segundo o protocolo do American College of Chest Physicians, a decisão deve ser individualizada e não é mencionada a dose da varfarina. ${ }^{1} \mathrm{O}$ American Heart Association é mais direto em recomendar o uso do cumarínico no segundo e terceiro trimestres, discutindo a sua segurança de forma relativa. Não há recomendação com relação à dose, mas estipula-se que o INR deve ficar entre 2,5 e 3,5. ${ }^{23}$

\section{Conclusão}

Existem atualmente protocolos bem-estruturados para a profilaxia de eventos trombóticos na gravideze para manejo das pacientes em situações especiais, como parto e puerpério. Infelizmente, apesar do surgimento de novas drogas antitrombóticas, ainda não temos uma opção que reúna eficácia, segurança e custo acessível a ser utilizada no período gestacional e durante o aleitamento. Com relação aos medicamentos disponíveis, atualmente, a heparina é a droga mais segura para ser utilizada no período gestacional, exceto nas pacientes portadoras de válvulas cardíacas metálicas que se beneficiam com o uso do cumarínico. Tanto os agentes antitrombóticos, quanto as novas drogas derivadas da heparina não contam com estudos suficientes que apontem segurança e eficácia para a sua utilização na gravidez e ficam reservadas para os casos de trombocitopania grave e intolerância ao uso de heparina.

\section{Referências}

1. Bates SM, Greer IA, Middeldorp S, Veenstra DL, Prabulos AM, Vandvik PO, et al. VTE, thrombophilia, antithrombotic therapy, and pregnancy: Antithrombotic Therapy and Prevention of Thrombosis, 9[th] ed: American College of Chest Physicians Evidence-Based Clinical Practice Guidelines. Chest. 2012;141(2 Suppl):e691S-736S.

2. American College of Obstetricians and Gynecologists Women's Health Care Physicians. ACOG Practice Bulletin No. 138: Inherited thrombophilias in pregnancy. Obstet Gynecol. 2013;122(3):706-17.

3. Cunningham FG. Williams obstetrics. 24[th] edition. ed. New York: McGraw-Hill Education/Medical; 2014. xviii, 1358 pages p.

4. Schaefer C, Hannemann D, Meister R, Eléfant E, Paulus W, Vial $\mathrm{T}$, et al. Vitamin $\mathrm{K}$ antagonists and pregnancy outcome. A multi-centre prospective study. Thromb Haemost. 2006;95(6):949-57.

5. Stevenson RE, Burton OM, Ferlauto GJ, Taylor HA. Hazards of oral anticoagulants during pregnancy. JAMA. 1980;243(15):1549-51.

6. Chan WS, Anand S, Ginsberg JS. Anticoagulation of pregnant women with mechanical heart valves: a systematic review of the literature. Arch Intern Med. 2000;160(2):191-6.

7. Levy RA, Jesús GR, Jesús NR. Obstetric antiphospholipid syndrome: still a challenge. Lupus. 2010;19(4):457-9.

8. Staico R, Vaz V, Cesar F, Feres F, Abizaid A, Mattos L, et al. Heparina Não-Fracionada e de Baixo Peso Molecular: Equivalência ou Superioridade na Intervenção Coronária Percutânea? Rev Bras Cardiol Invas. 2004;12(3):138-45.

9. Hirsh J, Guyatt G, Albers GW, Harrington R, Schünemann $\mathrm{HJ}$, American College of Chest Physician. Antithrombotic and thrombolytic therapy: American College of Chest Physicians Evidence-Based Clinical Practice Guidelines (8th Edition). Chest. 2008;133(6 Suppl):110S-2S.

10. Deruelle P, Coulon C. The use of low-molecular-weight heparins in pregnancy--how safe are they? Curr Opin Obstet Gynecol. 2007;19(6):573-7.

11. Empson M, Lassere M, Craig J, Scott J. Prevention of recurrent miscarriage for women with antiphospholipid antibody or lupus anticoagulant. Cochrane Database Syst Rev. 2005(2):CD002859.

12. Lindhoff-Last E, Kreutzenbeck HJ, Magnani HN. Treatment of 51 pregnancies with danaparoid because of heparin intolerance. Thromb Haemost. 2005;93(1):63-9.

13. Guyatt GH, Akl EA, Crowther M, Gutterman DD, Schuünemann HJ, American College of Chest Physicians Antithrombotic Therapy and Prevention of Thrombosis Panel. Executive summary: Antithrombotic Therapy and Prevention of Thrombosis, 9th ed: American College of Chest Physicians Evidence-Based Clinical Practice Guidelines. Chest. 2012;141(2 Suppl):7S-47S.

14. Tanimura K, Ebina Y, Sonoyama A, Morita H, Miyata S, Yamada $\mathrm{H}$. Argatroban therapy for heparin-induced thrombocytopenia during pregnancy in a woman with hereditary antithrombin deficiency. J Obstet Gynaecol Res. 2012;38(4):749-52.

15. Ekbatani A, Asaro LR, Malinow AM. Anticoagulation with 
Flávia C. dos Santos e cols. • Anticoagulação na gravidez

argatroban in a parturient with heparin-induced thrombocytopenia. Int J Obstet Anesth. 2010;19(1):82-7.

16. Young SK, AI-Mondhiry HA, Vaida SJ, Ambrose A, Botti JJ. Successful use of argatroban during the third trimester of pregnancy: case report and review of the literature. Pharmacotherapy. 2008;28(12):1531-6.

17. de Jesus GR, dos Santos FC, Oliveira CS, Mendes-Silva W, de Jesus NR, Levy RA. Management of obstetric antiphospholipid syndrome. Curr Rheumatol Rep. 2012;14(1):79-86.

18. Ruiz-Irastorza G, Crowther M, Branch W, Khamashta MA. Antiphospholipid syndrome. Lancet. 2010;376(9751):1498-509.

19. de Jesús GR, Rodrigues G, de Jesús NR, Levy RA. Pregnancy morbidity in antiphospholipid syndrome: what is the impact of treatment? Curr Rheumatol Rep. 2014;16(2):403.

20. de Jesus GR, Agmon-Levin N, Andrade CA, Andreoli L, Chighizola CB, Porter TF, et al. 14th International Congress on Antiphospholipid Antibodies Task Force report on obstetric an- tiphospholipid syndrome. Autoimmun Rev. 2014;13(8):795-813.

21. McLintock C. Anticoagulant choices in pregnant women with mechanical heart valves: balancing maternal and fetal risks-the difference the dose makes. Thromb Res. 2013;131 Suppl 1:S8-10.

22. Regitz-Zagrosek V, Blomstrom Lundqvist $C$, Borghi C, Cifkova $\mathrm{R}$, Ferreira R, Foidart JM, et al. ESC Guidelines on the management of cardiovascular diseases during pregnancy: the Task Force on the Management of Cardiovascular Diseases during Pregnancy of the European Society of Cardiology (ESC). Eur Heart J. 2011;32(24):3147-97.

23. Bonow RO, Carabello BA, Chatterjee K, de Leon AC, Faxon DP, Freed MD, et al. 2008 focused update incorporated into the ACC/AHA 2006 guidelines for the management of patients with valvular heart disease: a report of the American College of Cardiology/American Heart Association Task Force on Practice Guidelines. J Am Coll Cardiol. 2008;52(13):e1-142. 